\title{
Reply to "CLL and COVID-19 at the Hospital Clinic of Barcelona: an interim report" Analysis of six hematological centers in Lombardy
}

\author{
On behalf of CLL commission of Lombardy Hematology Network (REL)
}

\author{
Gianluigi Reda $\mathbb{D}^{1} \cdot$ Alessandro Noto ${ }^{1} \cdot$ Ramona Cassin $^{1} \cdot$ Giulia Zamprogna $^{2} \cdot$ Chiara Borella $^{3}$. Lydia Scarfó ${ }^{4}$. \\ Lucia Farina ${ }^{5} \cdot$ Alfredo Molteni ${ }^{6} \cdot$ Paolo Ghia ${ }^{4}{ }^{4} \cdot$ Alessandra Tedeschi $^{2} \cdot$ Marco Montillo $^{2} \cdot$ Rete Ematologica $^{2}$ \\ Lombarda (REL) Clinical Network ${ }^{7}$
}

Received: 22 May 2020 / Revised: 11 June 2020 / Accepted: 1 July 2020 / Published online: 4 August 2020

(c) Springer Nature Limited 2020

We read with interest the correspondence by Baumann et al. [1], reporting the first attempt to determine the prevalence of COVID-19 in the monocentric cohort of CLL patients at Hospital Clinic of Barcelona.

Four patients developed a mild symptomatic COVID-19 infection as of April 15, 2020, with a prevalence of $0.95 \%$ ( 4 of 420 pts). The authors speculated that the CLL-related immunodeficiency, rather than exacerbating the effects of SARS-CoV-2 might prevent them. Moreover they imply a potential role of immunomodulatory agents (e.g. ibrutinib) on the outcome of patients with CLL infected by SARSCoV-2 [2, 3].

The authors also refer to the recent recommendations for the management of cancer patients in COVID era published by the EHA Infectious Disease Scientific Working Group Executive Committee.

The panel suggested to consider treatment interruption in patients who experience community-acquired respiratory virus (CARV) infections, taking into account the type

Gianluigi Reda

gianluigi.reda@policlinico.mi.it

1 Hematology Unit, IRCCS Ca' Granda Ospedale Maggiore Policlinico of Milan, Milan, Italy

2 Department of Hematology, Niguarda Cancer Center, ASST Grande Ospedale Metropolitano Niguarda, Milan, Italy

3 Hematology Department, San Gerardo Hospital, ASST Monza (MB), Monza, Italy

4 Strategic Research Program on CLL, Università Vita-Salute San Raffaele and IRCCS Ospedale San Raffaele, Milano, Italy

5 Division of Hematology, Fondazione IRCCS Istituto Nazionale dei Tumori, Milano, Italy

6 Hematology and BMT Unit, ASST, Cremona, Italy

7 ASST Grande Ospedale Metropolitano Niguarda, Milan, Italy of therapy, its immunosuppressive potential and ability to produce severe lymphopenia.

For instance, treatment interruption is not mandatory for patients with controlled/chronic disease experiencing respiratory tract infectious disease while receiving regimens that are not associated with a clinically relevant immunosuppression [4].

To date, Italy is the 6th most affected country and Lombardy, accounting for about $1 / 6$ of the Italian population, has registered $37.5 \%$ of COVID-19 infections in the Italian territory.

Patients with hematological malignancies are believed to be more susceptible and have higher morbidity and mortality rates for infections than standard population. This is particularly evident in chronic lymphocytic leukemia (CLL), the most common type of leukemia in Western countries [5]. The reasons behind such higher infectious rates lead back to several coexisting factors. On the one hand, CLL is associated with a disease-related disruption of both humoral and cell-mediated immunity, on the other hand, patient-related factors such as age, disease stage, and type of treatment received play an important role [6].

Ibrutinib, an irreversible inhibitor of Bruton's tyrosine kinase (Btk) currently represents a milestone in the treatment of CLL and has been approved by FDA for the treatment of several B-cell malignancies and chronic graft-versus-host disease (cGVHD).

Btk is crucial for B-cell development and various B-cell functions, including natural antibody production. Interestingly, it is also expressed in numerous other cell lineages, including monocytes, macrophages, granulocytes, and dendritic cells [7].

With the aim to evaluate the impact of COVID-19 on CLL patients and particularly in those treated with ibrutinib, we collected data from six Hematology departments in Lombardy. 
We analyzed data from 2902 patients affected by CLL, of whom 278 on ibrutinib, 50 venetoclax, and 9 Idelalisib. Eighteen patients are currently receiving chemoimmunotherapy.

Among 2902 CLL patients, 23 cases of COVID infections have been reported with molecular testing for SARS-CoV-2. Of those, eight patients were receiving small molecules outside clinical trials. Fifteen patients were off-therapy: seven were treatment-naive, three had received fludarabine-based regimens, four bendamustine-rituximab, one patient had discontinued ibrutinib 1 year earlier.

Of patients treated with small molecules, four cases have been reported in patients on ibrutinib, three cases during venetoclax, and one patient while receiving idelalisib.

Focusing on ibrutinib patients, subjects were mainly males $(75 \%)$ with a median age at infection onset of 65 years (range 55-75). Two patients had no comorbidities, one presented hypertension and diabetes and one presented several comorbidities (hypertension, COPD, previous pulmonary embolism). All patients were previously treated for CLL (median 1.5 lines, range 1-3), median time on ibrutinib was 31 months (range 20-42 months) and none experienced dose reduction.

Considering clinical presentation of COVID-19, fever and cough were present at onset, and interstitial pneumonia was documented in all patients. Three of them developed ARDS with two requiring intubation; one was managed as an outpatient with hydroxychloroquine and azithromycin.

All hospitalized patients received hydroxychloroquine and steroids with one patient also receiving lopinavir/ ritonavir, and two were also treated with LMWH.

We observed one death in the ibrutinib group, in particular the most heavily treated patient carrying several comorbidities.

Despite the low number of events and SARS-CoV-2 infections in the ibrutinib group, we observed one case of fatal pneumonia in patients receiving ibrutinib. It is, of course, impossible to precisely quantify the number of COVID-positivity among asymptomatic/paucisymptomatic patients who did not report their symptoms and, therefore, might have tested positive on extended screening. However, all patients were contacted by phone during the study period to organize drug supply and investigate the presence of symptoms. It is tempting to hypothesize that ibrutinib may have prevented further clinical evolution toward a more severe course thanks to its modulating activity on cellmediated immune system.

In addition to its role on Btk, ibrutinib shows off-target inhibiting activity on IL-2-inducible T-cell kinase (Itk) and is thus capable of rebalancing the disease-inherent Th1/Th2 unbalance toward a Th1 phenotype in CLL patients.
Severe cases of SARS-CoV-2 pneumonia show an upward spike in inflammatory cytokines levels (particularly IL-6) with a concomitant decrease in absolute lymphocyte count and a relative $\mathrm{CD} 4 / \mathrm{CD} 8$ cells deficit compared with mild cases. The first pathological findings on alveolar exudates showed an imbalance in favor of Th17 compared to CD4 and CD8 cells. Th17 cells differentiate from Th0 after IL-6 stimulation, and are considered crucial in the genesis of SARS-CoV-2-related cytokine release syndrome (CRS) [8, 9].

Although the low numbers of our cohort make it impossible to draw further conclusions, the immune modulating activity of ibrutinib might give a biological rationale to the very low incidence (4/278) of COVID-positivity in this closely followed up group, possibly mitigating clinical manifestations of infection-driven CRS.

\section{Compliance with ethical standards}

Conflict of interest The authors declare that they have no conflict of interest.

Publisher's note Springer Nature remains neutral with regard to jurisdictional claims in published maps and institutional affiliations.

\section{References}

1. Baumann T, Delgado J, Montserrat E. CLL and COVID-19 at the hospital clinic of Barcelona: an interim report. Leukemia. 2020. https://doi.org/10.1038/s41375-020-0870-5.

2. Treon A, Steven P, Castillo J, Skarbnik AP, Soumerai JD, Ghobrial IM, et al. The BTK-inhibitor ibrutinib may protect against pulmonary injury in COVID-19 infected patients. Blood. 2020; 135:1912-5.

3. Weber ANR, Bittner Z, Liu X, Dang T-M, Radstac P, Brunner CI. Bruton's tyrosine kinase: an emerging key player in innate immunity. Front Immunol. 2017;8:1454.

4. von Lilienfeld-Toal M, Vehreschild JJ, Cornely O, Pagano L, Compagno F, EHA Infectious Disease Scientific Working Group, et al. Frequently asked questions regarding SARS-CoV2 in cancer patients-recommendations for clinicians caring for patients with malignant diseases. Leukemia. 2020;34:1487-94.

5. Morrison VA. Infectious complications of chronic lymphocytic leukaemia: pathogenesis, spectrum of infection, preventive approaches. Best Pract Res Clin Haematol. 2010;23:145-53.

6. Forconi F, Moss P. Perturbation of the normal immune system in patients with CLL. Blood. 2015;126:573-81.

7. Tillman BF, Pauff JM, Satyanarayana G, Talbott M, Warner JL. Systematic review of infectious events with the Bruton tyrosine kinase inhibitor ibrutinib in the treatment of hematologic malignancies. Eur $\mathbf{J}$ Haematol. 2018;100:325-34.

8. Zhang C, Wu Z, Li J, Zhao H, Wang G. The cytokine release syndrome (CRS) of severe COVID-19 and Interleukin-6 receptor (IL-6R) antagonist Tocilizumab may be the key to reduce the mortality. Int. J. Antimicrob. Agents. 2020;55:105954.

9. Miossec P, Kolls JK. Targeting IL-17 and TH17 cells in chronic inflammation. Nat Rev Drug Discov. 2012;11:763-76. 